\title{
Propagation of gravity waves through an SPH scheme with numerical diffusive terms
}

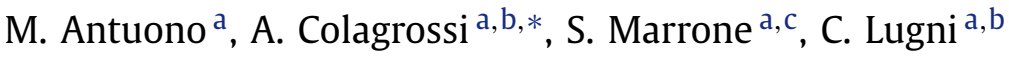 \\ a INSEAN, The Italian Ship Model Basin, Via di Vallerano 139, 00128 Rome, Italy \\ b CESOS: Centre of Excellence for Ship and Ocean Structures, NTNU, Trondheim, Norway \\ c Department of Mechanics and Aeronautics, University of Rome "Sapienza", Rome, Italy
}

\section{A R T I C L E I N F O}

\section{Article history:}

Received 8 July 2010

Received in revised form 23 November 2010

Accepted 2 December 2010

Available online 15 December 2010

\section{Keywords:}

Smoothed Particle Hydrodynamics

Weak-compressibility

Free-surface flows

Gravity wave propagation

Convergence tests

\begin{abstract}
A B S T R A C T
Basing on the work by Antuono et al. (2010) [1], an SPH model with numerical diffusive terms (here denoted $\delta$-SPH) is combined with an enhanced treatment of solid boundaries to simulate 2D gravity waves generated by a wave maker and propagating into a basin. Both regular and transient wave systems are considered. In the former, a large number of simulations is performed for different wave steepness and height-to-depth ratio and the results are compared with a BEM Mixed-Eulerian-Lagrangian solver (here denoted BEM-MEL solver). In the latter, the $\delta$-SPH model has been compared with both the experimental measurements available in the literature and with the BEM-MEL solver, at least until the breaking event occurs. The results show a satisfactory agreement between the $\delta$-SPH model, the BEMMEL solver and the experiments. Finally, the influence of the weakly-compressibility assumption on the $\mathrm{SPH}$ results is inspected and a convergence analysis is provided in order to identify the minimal spatial resolution needed to get an accurate representation of gravity waves.
\end{abstract}

(c) 2010 Elsevier B.V. All rights reserved.

\section{Introduction}

In the last years, the use of particle methods to simulate complex flow has been largely increased (see e.g. $[25,14]$ ). As a primary feature, those methods do not need a structured topological connection (grids) between the computational nodes. These nodes are treated as fluid particles followed during their motion, while their physical properties evolve in time according to the governing equations. One of the main advantages of such methods consists in the capability to deal with complex free-surface flows without an explicit enforcement of the dynamic condition along it (for details see [4]). In this context, the SPH scheme has widely proved to be an accurate and efficient solver to simulate violent flows with strong deformations of the free surface like dam-break flow impacts (see for example [2]).

Landrini et al. [14] proved that the SPH scheme can correctly simulate the near-shore bore propagation both in uniform depth and over a beach with constant slope. Notwithstanding that, the SPH literature beholds a few works dealing with the modeling of propagation of gravity waves (see, for example, [13]).

In the present work we use a modified version of the weaklycompressible SPH scheme with diffusive terms described in An-

\footnotetext{
* Corresponding author at: INSEAN, The Italian Ship Model Basin, Via di Vallerano 139, 00128 Rome, Italy. Tel.: +3906 50299 343; fax: +3906 5070619 .

E-mail addresses: m.antuono@insean.it (M. Antuono), a.colagrossi@insean.it (A. Colagrossi), s.marrone@insean.it (S. Marrone), c.lugni@insean.it (C. Lugni).
}

tuono et al. [1] (hereinafter denoted by $\delta$-SPH) to study the propagation of $2 \mathrm{D}$ gravity waves generated by a wave maker into a $2 \mathrm{D}$ wave basin. In contrast with the numerical scheme proposed in [1], the present SPH scheme contains a diffusive term only in the continuity equation while the influence of the internal energy is neglected. This idea was firstly proposed by Molteni and Colagrossi [24] and, using a different approach, by Ferrari et al. [10] where a Rusanof-type flux was added inside the continuity equation. In the present SPH scheme, a linear state equation with a constant sound velocity is used in order to simplify the numerical model. The solid boundaries and the wave maker are modeled through the fixed ghost particle technique described in [3]. Then, the present work has to be regarded as a further validation of this numerical technique. For the results presented in this work a heuristic convergence analysis is provided.

In the first part of the paper, the evolution of a standing wave in a rectangular tank is studied in order to measure the dissipation of the proposed scheme. The dissipation is mainly caused by the use of a numerical viscous term in the SPH scheme. This term, widely adopted in the SPH literature (see for example [25]), makes the numerical scheme stable. Although it is not used to simulate a viscous fluid, the numerical viscous term approximates the physical viscous term of an incompressible fluid, i.e. the Laplacian of the fluid velocity (see, for example, [9]). The boundary condition on rigid boundaries is a free slip condition. As a consequence, in physical problems where the solid boundary effects are negligible (like, for example, the propagation of gravity waves), the dissipative rate 
predicted by the numerical scheme is close to the physical one. Specifically, we use the approximate analytical formula derived by Lighthill [17] as a validation for the numerical scheme. This formula gives an estimation of the damping of gravity waves caused by viscous effects and is particularly suited for comparison with the SPH solutions since it has been derived under the assumption that the boundary layer dissipation is negligible. To this purpose, the dissipation rate predicted by the SPH scheme is generally in good agreement with the prediction of the approximate analytical formula. Anyway, the standing wave problem allows an accurate inspection of a further problem which arises when the wave amplitude is too small. An overdamping is observed when the wave amplitude is lower than the initial particle spacing, $d x$. This behavior is related to spurious numerical effects and is likely caused by the inability of the numerical scheme to represent the wave evolution when the spatial resolution is too coarse. In any case, the analysis of this phenomenon (further inspected in the regular wave simulations) allows the setting of the limits of applicability of the SPH scheme to study the gravity waves propagation.

In the second part of the paper, the propagation of regular wave trains is used to validate the proposed SPH scheme. A large number of test cases are considered to emphasize the nonlinear features of the wave propagation. Both the wave steepness and the wave height-to-depth ratios spreading from deep to shallow water are considered. The SPH results are compared with the predictions of the Mixed-Eulerian-Lagrangian Boundary Element Method (hereinafter BEM-MEL) developed in [20]. The BEM-MEL solver, based on the potential theory (that is, inviscid and irrotational flows), is the most appropriate scheme to describe the propagation of gravity waves (see for example $[18,19,12,5,6,26])$ at least until the closure of the breaking wave. Because of the limitations of the potential flow theory, the wave height of the regular wave systems are chosen to emphasize the nonlinear features without inducing a breaking events. In this way, the capability of the SPH scheme (generally implemented with the use of an artificial viscosity) to approximate inviscid fluids is fully inspected.

Although the BEM-MEL solver is generally faster and more accurate than the SPH schemes in modeling small gravity waves, it is limited to the analysis of non-breaking waves. On the contrary, due to its Lagrangian structure, the SPH solvers are among the best codes to model violent breaking events. In any case, since the breaking is generally preceded by a long non-breaking evolution, the validation of the present $\delta$-SPH scheme through non-breaking wave problems is of fundamental importance.

Finally, the last part of the paper focuses on the propagation of wave packets. A wave packet is given by the interaction of several wave components whose amplitudes are chosen in a prescribed frequency spectrum. The corresponding wave phases are calculated to realize their focusing in a prescribed position of the wave basin (i.e. the focusing point) and at a fixed time instant. Because of its nature, the wave packet is a good candidate to check the nonlinear propagation of a multicomponent wave train. Following Dommermuth et al. [7], the wave packet is used to get a breaking wave at the focusing point and the numerical results are then compared with the experimental data provided in [7].

\section{The $\delta$-SPH scheme}

In the SPH method, the fluid domain $\Omega$ is discretized in a finite number of particles representing elementary fluid volumes $d V$, each one with its own local mass $\mathrm{dm}$ and other physical properties. In this context, a generic field $f$ at the position $\boldsymbol{r}_{i}$ of the $i$-th particle is approximated through the convolution sum

$\langle f\rangle\left(\boldsymbol{r}_{i}\right)=\sum_{j} f_{j} W\left(\boldsymbol{r}_{i}-\boldsymbol{r}_{j}, h\right) d V_{j}$ where $f_{j}$ is the value of $f$ associated to the generic particle $j, d V_{j}$ is its volume and finally $W\left(\boldsymbol{r}_{i}-\boldsymbol{r}_{j}, h\right)$ is a kernel function with a finite support. The kernel function is defined so that the integral on its support is equal to one. The symbol $h$ is a reference length of this support and is generally called smoothing length. When $h$ goes to zero the kernel function $W$ becomes a delta Dirac "function". For the ease of notation, hereinafter we denote $W\left(\boldsymbol{r}_{i}-\boldsymbol{r}_{j}, h\right)$ simply through $W\left(\boldsymbol{r}_{j}\right)$. The choice of the kernel function affects both the CPU requirements and the stability properties of the algorithm. In this work a Gaussian kernel with a compact support has been adopted (see, for example, $[24,14]$ ). In all the simulations presented in this paper the particles are initially set on a regular lattice, the initial particles' distance is $d x$ and therefore the initial volume of the particle is $d V=d x^{2}$. As reported in [15], the value of the ratio $h / d x$ is generally chosen by following the practical rule that a linear field has to be well reproduced when the particles are distributed on a Cartesian uniform lattice. Using $h / d x=4 / 3$ and the adopted kernel, this rule is satisfied with an error of order of $10^{-4}$. The spatial derivatives of the field $f$ can be estimated using the formula (1)

$\langle\nabla f\rangle\left(\boldsymbol{r}_{i}\right)=\sum_{j}(\nabla f)_{j} W\left(\boldsymbol{r}_{j}\right) d V_{j}$.

Following [4], the previous formula can be approximated by

$\langle\nabla f\rangle\left(\boldsymbol{r}_{i}\right)=\sum_{j} f_{j} \nabla_{i} W\left(\boldsymbol{r}_{j}\right) d V_{j}+\left\langle I_{\partial} \Omega\right\rangle_{i}$

where the surface term $\left\langle I_{\partial \Omega}\right\rangle_{i}$ has to be handled in an adequate way in order to accurately account for the considered boundary conditions, as shown in [4].

Most of the SPH schemes is based on the assumption of barotropic and weakly-compressible fluid. Then, the reference equations for the flow evolution are the Euler equations:

$$
\frac{D \rho}{D t}=-\rho \nabla \cdot \boldsymbol{u}, \quad \rho \frac{D \boldsymbol{u}}{D t}=-\nabla p+\rho \boldsymbol{f}, \quad p=c_{0}^{2}\left(\rho-\rho_{0}\right),
$$

where $\rho, p$ and $\boldsymbol{u}$ are the density, pressure and velocity fields, respectively, $\boldsymbol{f}$ is the body force field, $\rho_{0}$ the density at the free surface and $c_{0}$ the sound velocity. Differently from [1], in the present work we use a linear state equation since we do not deal with violent fluid-structure interactions.

To reduce the computational effort, most of the weaklycompressible SPH solvers imposes the sound speed much lower than the physical one, however, one order of magnitude larger than the maximum velocity of the investigated flow. Anyway, in the case of gravity waves the flow velocity is generally small while the most important quantity associated with the wave propagation is the wave celerity, that is, $c^{2}=g / k \tanh (k H)$ where $H$ is the still water depth, $k$ is the wave number and $g$ the gravity acceleration (see, for example, [22]). For $k H$ going to zero, the shallow water regime is approached and the relation above becomes $c^{2}=g H$. Since the latter expression is an upper bound for the wave celerity (that is $g / k \tanh (k H) \leqslant g H$ ), we choose $c_{0}=10 \sqrt{g H}$.

When the system (4) is written in the SPH formulation, an artificial viscous term is generally added in the momentum equation for stability reasons (see for example [25]). Here the SPH scheme proposed by Antuono et al. [1] is used: a proper artificial diffusive term is added into the continuity equation in order to remove the spurious numerical high-frequency oscillations in the pressure field. Further, the XSPH velocity correction is used to regularize the velocity field (see for example [25]). Then, the discrete $\delta$-SPH scheme reads: 


$$
\left\{\begin{aligned}
& \frac{D \rho_{i}}{D t}=-\rho_{i} \sum_{j}\left(\boldsymbol{u}_{j}-\boldsymbol{u}_{i}\right) \cdot \nabla_{i} W\left(\boldsymbol{r}_{j}\right) d V_{j} \\
&+\delta h c_{0} \sum_{j} \psi_{i j} \cdot \nabla_{i} W\left(\boldsymbol{r}_{j}\right) d V_{j}, \\
& \rho_{i} \frac{D \boldsymbol{u}_{i}}{D t}=-\sum_{j}\left(p_{j}+p_{i}\right) \nabla_{i} W\left(\boldsymbol{r}_{j}\right) d V_{j}+\rho_{i} \boldsymbol{f}_{i} \\
&+\alpha h c_{0} \rho_{0} \sum_{j} \pi_{i j} \nabla_{i} W\left(\boldsymbol{r}_{j}\right) d V_{j}, \\
& \frac{D \boldsymbol{r}_{i}}{D t}= \boldsymbol{u}_{i}+\varepsilon_{X} \sum_{j}\left(\boldsymbol{u}_{j}-\boldsymbol{u}_{i}\right) W\left(\boldsymbol{r}_{j}\right) d V_{j}, \\
& p_{i}=c_{0}^{2}\left(\rho_{i}-\rho_{0}\right),
\end{aligned}\right.
$$

where:

$\boldsymbol{\psi}_{i j}=2\left(\rho_{j}-\rho_{i}\right) \frac{\boldsymbol{r}_{j i}}{\left|\boldsymbol{r}_{i j}\right|^{2}}-\left[\langle\nabla \rho\rangle_{i}^{L}+\langle\nabla \rho\rangle_{j}^{L}\right]$,

$\pi_{i j}=\frac{\left(\boldsymbol{u}_{j}-\boldsymbol{u}_{i}\right) \cdot \boldsymbol{r}_{j i}}{\left|\boldsymbol{r}_{i j}\right|^{2}}$,

and $\boldsymbol{r}_{i j}=-\boldsymbol{r}_{j i}=\boldsymbol{r}_{i}-\boldsymbol{r}_{j}$. The symbol $\langle\nabla \rho\rangle_{i}^{L}$ indicates the renormalized gradient defined in [16]. The symbols $\rho_{i}, p_{i}$ and $\boldsymbol{u}_{i}$ denote the $i$-th particle density, pressure and velocity, respectively. The coefficient $\delta$ determines the order of magnitude of the diffusive term, $\alpha$ the order of magnitude of the artificial viscous term and $\varepsilon_{X}$ the influence of the XSPH correction on the actual velocity field. Here, $\varepsilon_{X}=0.25, \delta=0.1$ and $\alpha$ is chosen in the range [0.01, 0.05].

The system (5) preserves the global mass and both the linear and angular momenta. Moreover, the diffusive term in the continuity equation, the artificial viscosity and the XSPH correction go to zero as the spatial resolution increases (i.e. when $h$ goes to zero), recovering the consistency with the Euler equations.

The numerical viscous term added inside the momentum equation approximates the Laplacian of the velocity for incompressible fluids (see [9]). Notwithstanding that, a free slip condition is implemented along the solid boundary since in the propagation of gravity waves the boundary layer effects are negligible. The corresponding numerical kinematic viscosity is theoretically given in two dimension by

$v=\alpha c_{0} h / 8=\alpha c_{0} d x / 6$

(see e.g. [25]). For the gravity wave problem, a value of alpha larger than 0.01 is used in order to ensure the stability of the numerical algorithm. This choice is a limitation of the SPH scheme since the artificial viscosity damps out largely the gravity waves for long time simulations. An estimation of the damping effects is discussed in Section 3 making the results of this paper useful for the comparison with other numerical solvers.

\section{The solid boundary treatment}

The enhanced treatment of the solid boundaries proposed in [3] and [21] is used here to deal with the solid boundary at the wave maker, the tank walls and the bottom. The former is a rigid boundary moving with a prescribed time law. Within the SPH scheme the modeling of moving solid boundaries with a generic shape is not a trivial matter. Anyway, similarly to [3], in the present work we implement an enhanced treatment of solid boundaries based on the use of fixed ghost particles. This method generally provides a simple and accurate procedure for the description of moving solid profiles and can be regarded as a generalization of the classical ghost-particle method widely used in the SPH literature (see for example [2]).

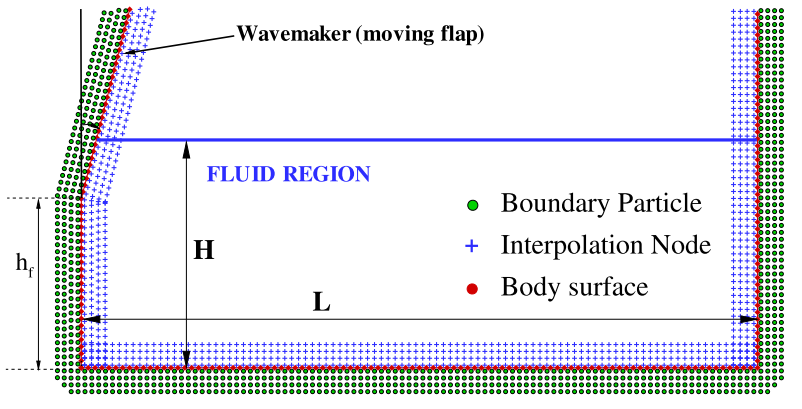

Fig. 1. Sketch of the fixed ghost particles near the wave maker. The symbol $h_{f}$ indicates the distance of the flap hinge from the tank bottom.

In the standard ghost particle technique at each time step any particle nearby the solid boundary is mirrored into a ghost with respect to that boundary. In contrast, here the ghost particles are fixed in the frame of reference of the body. To compute the quantities attributed to each ghost particle, an interpolation point is associated to it. This interpolation point is obtained by mirroring the position of the fixed ghost particle into the fluid domain, see Fig. 1 .

The algorithm to generate the fixed ghost particles is detailed in the following: (a) the tank profile is approximated by regular equispaced body nodes with a prescribed distance $d s$; (b) the normal and the tangent unit vectors are computed, assuming the normal vectors to point outwards the fluid domain; (c) using the normal vector, the body nodes are moved outwards the fluid at a distance $d s / 2$ from the body profile; (d) a new profile is generated along which equispaced nodes with distance $d s$ are set. Such a profile represents an expansion of the tank profile and the nodes along it are the fixed ghost particles. In the same way but using a vector opposite to the normal, each fixed ghost particle is associated with an interpolation point inside the fluid. The procedure is repeated to cover the interaction radius of the fluid particles (see Fig. 1). In case of singular points along the tank profile (like the hinge of a flap wave maker), the procedure described above is applied starting on both sides of the singularity. The generation of the fixed ghost particles is repeated at each time step to update the position of the wave maker.

The main advantage of the fixed ghost particle technique with respect to the classic ghost particles consists in a uniform distribution of the fixed ghost, independently from the position of the fluid particles. This allows a simple modeling of complex 2D geometries. Further, the use of a Moving Least Square interpolator (see [11] for more details) ensures an accurate mirroring procedure of the flow quantities.

In this work only the free-slip boundary condition is prescribed for the solid boundaries. This condition is enforced keeping the tangential component of velocity unaltered during the mirroring procedure while the normal component of the fluid velocity is reversed to prevent particles going through the solid boundary. For moving boundaries the velocities components have to be considered with respect to the frame of reference attached to the boundary. To assign the pressure field along the solid boundaries, the Neumann boundary condition is enforced, that is:

$$
\frac{\partial p}{\partial n}=-\rho\left[\frac{D \boldsymbol{u}_{\boldsymbol{b}}}{D t} \cdot \boldsymbol{n}+\left(\boldsymbol{u}_{\boldsymbol{b}}-\boldsymbol{u}\right) \cdot\left(\boldsymbol{\omega}_{\boldsymbol{b}} \times \boldsymbol{n}\right)-\boldsymbol{f} \cdot \boldsymbol{n}\right],
$$

where $\boldsymbol{n}$ is the normal unit vector to the solid profile, $\boldsymbol{u}_{\boldsymbol{b}}$ is the velocity of the solid boundary and $\omega_{b}$ is its angular velocity. Note that (7) is obtained from the momentum equation using a free slip condition along the solid profiles. In the case of no-slip conditions, it is sufficient to neglect the term containing $\boldsymbol{\omega}_{\boldsymbol{b}}$. For more details we address the reader to Doring [8]. 

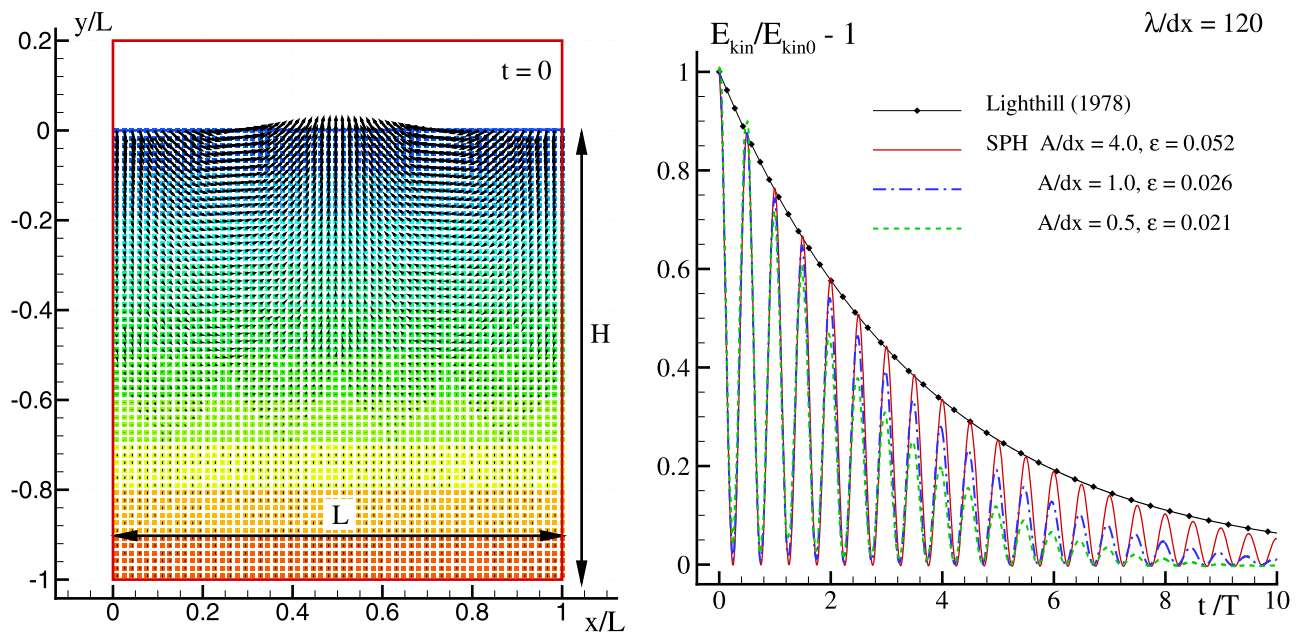

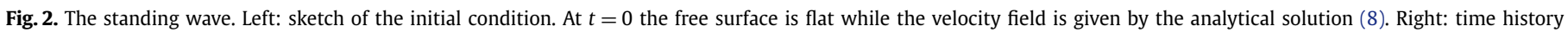
of the total kinetic energy ( $\beta=0.1097$ ).

\section{Test cases}

\subsection{Standing waves}

Before dealing with the generation and propagation of gravity waves, we study the evolution of a standing wave in a $2 D$ squared tank with length $L$. For an inviscid fluid, the linear theory predicts that the standing wave evolves infinitely without any change of amplitude, i.e. the total energy is conserved. Similarly, the mean over the wave period of the kinetic energy maintains constant during the evolution. On the contrary, the SPH do not preserve the energy because of the numerical viscous term inside the momentum equation. As a consequence, for long time evolution the mean kinetic energy goes to zero and the standing wave is completely damped. For these reasons, this problem is used to get a measure of the numerical damping of the proposed SPH scheme through the analysis of the kinetic energy time history. In this way it is possible to check whether the numerical dissipation is mainly due to the artificial viscosity (see Eq. (6)) or to other numerical effects, identifying the limits of applicability of the SPH scheme.

For the standing wave problem, we fixed the water filling height to $H=L$. In the following $\lambda$ indicates the wave length of the standing wave and $k$ the corresponding wave number (i.e. $k=2 \pi / \lambda) ; A$ is the amplitude of the standing wave and $\epsilon$ denotes the ratio $2 A / H$. For small-amplitude waves (i.e. small $\epsilon$ ), the potential theory gives the following approximate analytical solution:

$\varphi(x, y, t)=\varphi_{0}(x, y) \cos (\omega t)$;

$\varphi_{0}(x, y)=-\epsilon \frac{H g}{2 \omega} \frac{\cosh [k(y+H)]}{\cosh (k H)} \cos (k x)$.

The period of oscillation of the standing waves is $T=2 \pi / \omega$ where the circular frequency $\omega$ is given by the dispersion relation: $\omega^{2}=$ $g k \tanh (k H)$. The frame of reference is indicated by the sketch in the left plot of Fig. 2. At time $t=0$ the free surface is horizontal and the time derivative of the velocity potential $\dot{\varphi}$ is zero in the whole domain, therefore the pressure field can be simply assumed to be hydrostatic with an error of $\mathcal{O}\left(\epsilon^{2}\right)$. By definition the initial fluid velocity is given by $\nabla \varphi_{0}$ (see left plot of Fig. 2). Since the SPH scheme contains a numerical viscosity term (see Eq. (5)), its solution converges to (8) only for $h$ going to zero. For finite values of $h$ and neglecting the boundary layer dissipation, an analytical expression of the energy dissipation can be derived following the procedure described by Lighthill [17]. Under the assumption made in [17], the time evolution of total kinetic energy is given by the following approximate solution:
$E_{\text {kin }}(t)=\epsilon^{2} g \frac{\lambda H^{2}}{32} e^{-4 v k^{2} t}[1+\cos (2 \omega t)]$

where the numerical kinematic viscosity given by Eq. (6) is used to compute $\nu$. The damping coefficient $\beta=4 \nu k^{2}$ depends on the wave number and on the numerical kinematic viscosity.

The dissipation predicted by (9) is due to the numerical viscous term included into the momentum equation of the SPH scheme. Anyway, a larger damping is observed when the spatial resolution is too coarse. This phenomenon, related to numerical approximations, has to be avoided because the SPH solver is working out of its limits of applicability.

The overdamping of the standing wave is displayed in the right panel of Fig. 2. Here, $\lambda=L$ ( $L$ is the tank width), the spatial resolution is fixed (i.e. $d x$ constant) and three different wave heights are considered (i.e. three different values of $A / d x$ ). Since $\alpha=0.05$, the damping rate predicted by (9) is constant (that is, $\beta=0.1097$ ). Anyway, for $A / d x=0.5$ and $A / d x=1$, the dissipation shown by the SPH solver is much larger and a good agreement is obtained only for $A / d x=4$. This means that the SPH cannot properly model gravity waves whose height is of the same order of magnitude of the mean particle distance (that is, $A / d x \lesssim 1$ ).

Apart from this, a good agreement is observed when $A / d x>2$. This is, for example, highlighted in Fig. 3. Here, the wavelength, the wave height and the nonlinearity parameter are kept constant and the spatial resolution increases. Since $\alpha=0.05$, each numerical simulations is characterized by a different dissipation rate (i.e. a different value of $\beta$ ). The most viscous case, i.e. $\beta=0.2193$, is damped in only 10 periods; in contrast, the simulation with $\beta=0.0274$ just halves in the same time interval. The fair match between the analytical solution (9) and the SPH output is a clear indication that for this test case the numerical damping is only related to the artificial viscosity dissipation. As a consequence, the solid boundary treatment adopted, the weakly-compressible assumption and the use of diffusive term in the continuity equation do not introduce any significant error. The simulation has been repeated using different time integrators (see e.g. [24]) recovering the same solution plotted in Fig. 3.

\subsection{Regular waves}

Here we focus on the propagation of regular waves postponing the analysis of the wave packet to the next section. In all the SPH simulations a uniform spatial resolution is used. This is not an optimal choice because the use of a finer spatial resolution near the free surface and a coarser one as the depth increases 

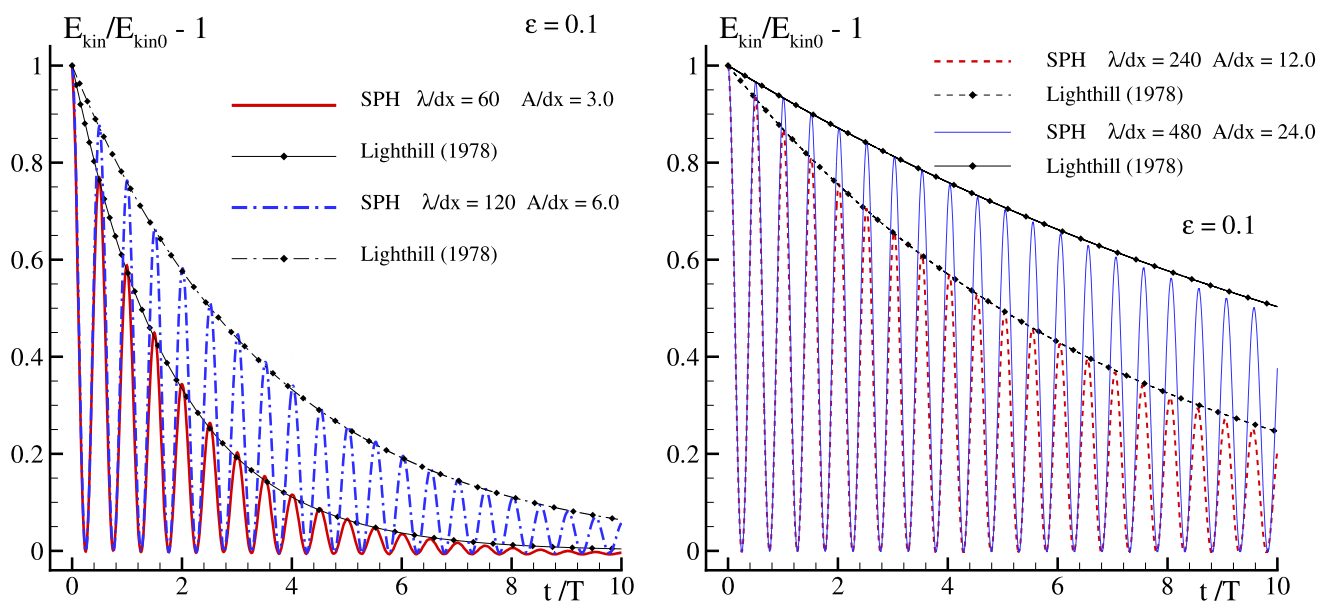

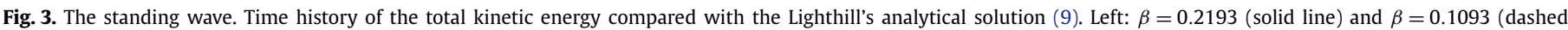
line). Right: $\beta=0.0545$ (dashed line) and $\beta=0.0274$ (solid line).

Table 1

Test case matrix for regular waves in deep water regime.

\begin{tabular}{|c|c|c|c|c|c|c|c|c|c|}
\hline & \multicolumn{9}{|c|}{ Deep water } \\
\hline & $\lambda / H$ & $\mu$ & $\epsilon$ & $\gamma$ & $N p$ & $H / d x$ & $\lambda / d x$ & $A / d x$ & $\beta T$ \\
\hline DW_01 & 2.00 & 3.14 & 0.03 & 0.05 & $404 K$ & 130 & 260 & 1.95 & $1.80 \times 10^{-2}$ \\
\hline DW_02 & 2.00 & 3.14 & 0.07 & 0.11 & $404 K$ & 130 & 260 & 4.55 & $1.80 \times 10^{-2}$ \\
\hline DW_03 & 2.00 & 3.14 & 0.13 & 0.20 & $404 K$ & 130 & 260 & 8.45 & $1.80 \times 10^{-2}$ \\
\hline DW_04 & 2.00 & 3.14 & 0.23 & 0.36 & $468 K$ & 150 & 260 & 17.3 & $1.56 \times 10^{-2}$ \\
\hline DW_05 & 1.40 & 4.49 & 0.04 & 0.09 & $538 K$ & 150 & 210 & 3.00 & $2.66 \times 10^{-2}$ \\
\hline DW_06 & 1.40 & 4.49 & 0.06 & 0.13 & $538 K$ & 150 & 210 & 4.50 & $2.66 \times 10^{-2}$ \\
\hline DW_07 & 1.00 & 6.28 & 0.01 & 0.04 & $538 K$ & 150 & 150 & 0.75 & $4.40 \times 10^{-2}$ \\
\hline DW_08 & 1.00 & 6.28 & 0.03 & 0.09 & $538 K$ & 150 & 150 & 2.25 & $4.40 \times 10^{-2}$ \\
\hline DW_09 & 1.00 & 6.28 & 0.05 & 0.17 & $314 K$ & 150 & 150 & 3.75 & $4.40 \times 10^{-2}$ \\
\hline
\end{tabular}

Table 2

Test case matrix for regular waves in intermediate water regime.

\begin{tabular}{|c|c|c|c|c|c|c|c|c|c|}
\hline & \multicolumn{9}{|c|}{ Intermediate water } \\
\hline & $\lambda / H$ & $\mu$ & $\epsilon$ & $\gamma$ & $N p$ & $H / d x$ & $\lambda / d x$ & $A / d x$ & $\beta T$ \\
\hline IW_01 & 12.0 & 0.52 & 0.22 & 0.06 & $223 K$ & 40 & 480 & 4.40 & $5.72 \times 10^{-3}$ \\
\hline IW_02 & 12.0 & 0.52 & 0.34 & 0.09 & $223 K$ & 40 & 480 & 6.80 & $5.72 \times 10^{-3}$ \\
\hline IW_03 & 7.40 & 0.85 & 0.04 & 0.04 & $265 K$ & 60 & 444 & 1.20 & $6.57 \times 10^{-3}$ \\
\hline IW_04 & 7.40 & 0.85 & 0.23 & 0.20 & $265 K$ & 60 & 444 & 6.90 & $6.57 \times 10^{-3}$ \\
\hline IW_05 & 6.00 & 1.05 & 0.11 & 0.06 & $356 K$ & 50 & 300 & 2.75 & $1.02 \times 10^{-2}$ \\
\hline IW_06 & 6.00 & 1.05 & 0.25 & 0.13 & $356 K$ & 50 & 300 & 6.25 & $1.02 \times 10^{-2}$ \\
\hline IW_07 & 3.00 & 2.09 & 0.11 & 0.11 & $514 K$ & 60 & 180 & 3.30 & $2.15 \times 10^{-2}$ \\
\hline IW_08 & 3.00 & 2.09 & 0.22 & 0.23 & $514 K$ & 60 & 180 & 6.60 & $2.15 \times 10^{-2}$ \\
\hline
\end{tabular}

strongly reduces the CPU cost (see for example [3]). Anyway, since the present work is a validation of the $\delta$-SPH scheme, we preferred to limit the numerical variables/parameters. For the same reason, we did not use any beach or damping technique to reduce wave reflection at the end walls of the tank. As a consequence, the tank length is set to simulate about 12 wave periods without any reflection at the right end wall. This time of simulation is generally too short to reach a steady-state solution and, therefore, only the transient evolution is considered. A longer time interval would require an extension of the tank and a large increase of the particle number and of the computational cost. In any case, the comparison between different numerical solvers is generally more demanding during the transient evolution than during the steady-state. For the detection of the free-surface, we used the algorithm described in Marrone et al. [23].

Tables 1, 2 and 3 represent the main parameters of the test cases considered in the present work. Specifically, $\lambda$ is the wave length, $\mu=2 \pi H / \lambda$ is the parameter accounting for the dispersive effects, $\epsilon=2 A / H$ is the nonlinearity parameter ( $A$ is the wave amplitude) and $\gamma=2 \pi A / \lambda$ is the wave steepness. To allow a comparison with the MEL-BEM solver, test cases are split in deep-water ( $\mu>3$, see Table 1$)$, intermediate-water $(0.3<\mu \leqslant 3$, see Table 2$)$ and shallow-water $(\mu \leqslant 0.3$, see Table 3$)$ regime. The parameters $\epsilon$ and $\gamma$ are chosen to avoid the occurrence wave breaking, but for the case DW_04 where the leading crest is breaking. The wave maker is a piston for $\lambda \geqslant 12$ while a flap has been adopted for the remaining cases (see Fig. 1). The flap paddle is hinged at the tank bottom with the exception of the cases DW_05, DW_06 (hinged at 0.3 from the tank bottom) and DW_07, DW_08 (hinged at 0.5 from the tank bottom). In all the cases a sinusoidal time law has been imposed to the paddle. Further, a ramp has been used in some test cases during the initial stage of the evolution to avoid impulsive transitory. Details on the wave maker motion and on the ramp are given in Appendix A.

In Tables 1-3 are also reported the parameters related to the spatial resolutions used in the SPH simulations: the total number of particles $N_{p}$, the number of particles along the depth (i.e. $H / d x$ ) and the ratios $\lambda / d x, A / d x$, i.e. the number of particle for wave- 
Table 3

Test case matrix for regular waves in shallow water regime.

\begin{tabular}{|c|c|c|c|c|c|c|c|c|c|}
\hline & \multicolumn{9}{|c|}{ Shallow water } \\
\hline & $\lambda / H$ & $\mu$ & $\epsilon$ & $\gamma$ & $N p$ & $H / d x$ & $\lambda / d x$ & $A / d x$ & $\beta T$ \\
\hline SW_01 & 30.0 & 0.21 & 0.12 & 0.01 & $400 K$ & 40 & 1200 & 2.40 & $2.21 \times 10^{-3}$ \\
\hline SW_02 & 30.0 & 0.21 & 0.27 & 0.03 & $144 K$ & 20 & 600 & 2.70 & $4.42 \times 10^{-3}$ \\
\hline SW_03 & 30.0 & 0.21 & 0.39 & 0.08 & $400 K$ & 40 & 1200 & 7.80 & $2.21 \times 10^{-3}$ \\
\hline SW_04 & 25.0 & 0.25 & 0.13 & 0.02 & $400 K$ & 40 & 1000 & 2.60 & $2.66 \times 10^{-3}$ \\
\hline SW_05 & 25.0 & 0.25 & 0.25 & 0.03 & $144 K$ & 20 & 500 & 2.50 & $5.32 \times 10^{-3}$ \\
\hline SW_06 & 25.0 & 0.25 & 0.44 & 0.11 & $400 K$ & 40 & 1000 & 8.80 & $2.66 \times 10^{-3}$ \\
\hline SW_07 & 21.0 & 0.30 & 0.13 & 0.02 & $144 K$ & 20 & 420 & 1.30 & $6.36 \times 10^{-3}$ \\
\hline SW_08 & 21.0 & 0.30 & 0.22 & 0.03 & $144 K$ & 20 & 420 & 2.20 & $6.36 \times 10^{-3}$ \\
\hline SW_09 & 21.0 & 0.30 & 0.36 & 0.11 & $144 K$ & 20 & 420 & 3.60 & $6.36 \times 10^{-3}$ \\
\hline
\end{tabular}

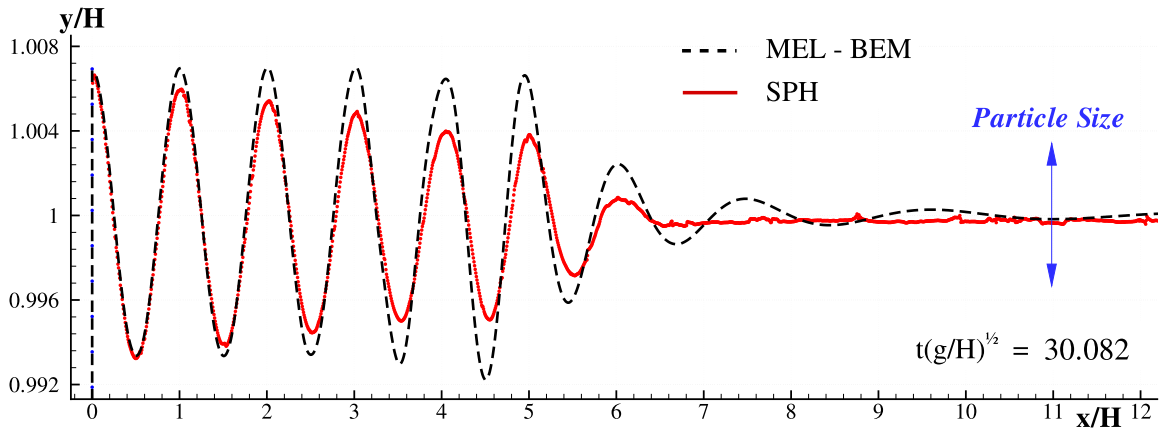

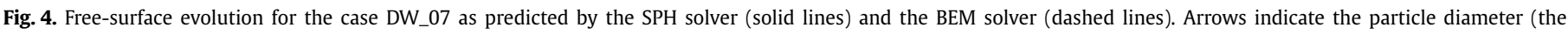
length is referred to the $y$-axis). The SPH wave is highly damped by the artificial viscosity.
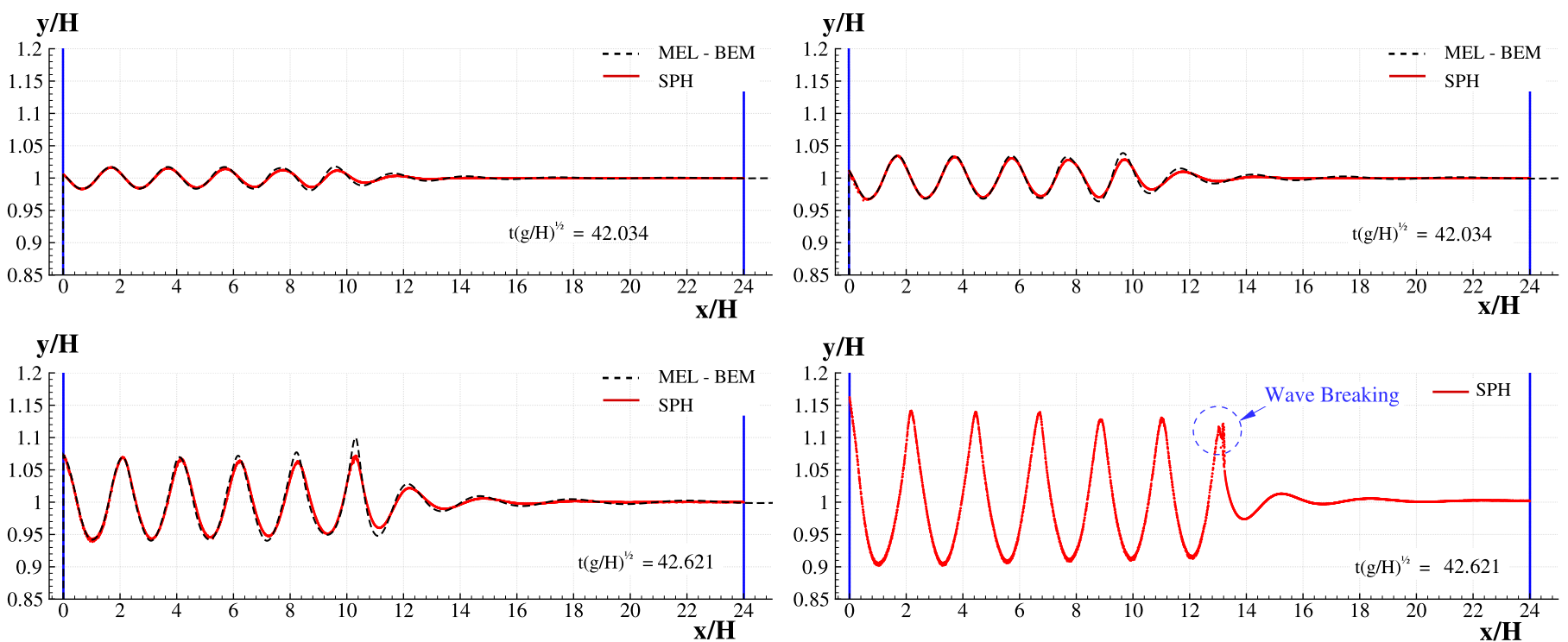

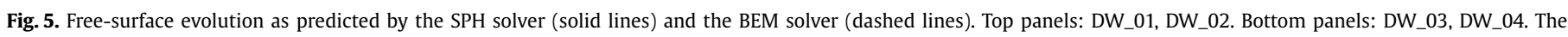
wave length of these cases is $\lambda / H=2$ while the steepness increases from $\gamma=0.05$ up to $\gamma=0.36$.

length and wave amplitude, respectively. The last columns contain the parameter $\beta T$ where $\beta$ is the damping coefficient defined in Section 3.1 and $T$ is the wave period. Last parameter gives an estimate of the damping per period caused by the numerical viscosity of the SPH scheme.

Basing on the results of Section 3.1, a wave amplitude $A$ greater than $d x$ has been chosen for almost all simulations of regular waves (the only exception is the case DW_07 which is discussed in the following). This choice ensures a small dissipation due to spurious numerical effects of the SPH solver. Further, to get an accurate enough representation of the wave propagation phenomenon, a spatial discretization $\lambda / d x>100$ is chosen. In the BEM-MEL solver, a spatial discretization $\lambda / d x=90$ is used while no limits exist for wave amplitude. The deep water case DW_07 has the lowest ratio $A / d x$, set equal to 0.75 . The free surface profile at time $t \sqrt{g / H}=30.08$, evaluated by the two solvers SPH (solid line) and BEM-MEL (dashed line), is reported in Fig. 4. The damped behavior of the wave in the SPH solution resembles the one observed in the previous section for the standing wave when $A / d x<1$. In contrast, the case DW_03 using ratio $A / d x$ equal to 8.45 shows a much reduced wave damping (see right-top plot of Fig. 6).

In Fig. 5 (from left to right and from top to bottom), we show the comparison of the free surface evolution as predicted by the $\delta$-SPH solver (solid lines) and the BEM solver (dashed lines) for the deep-water cases DW_01, DW_02, DW_03, DW_04 (reported in Table 1 ). These cases are characterized by a wave length $\lambda / H=2$ 


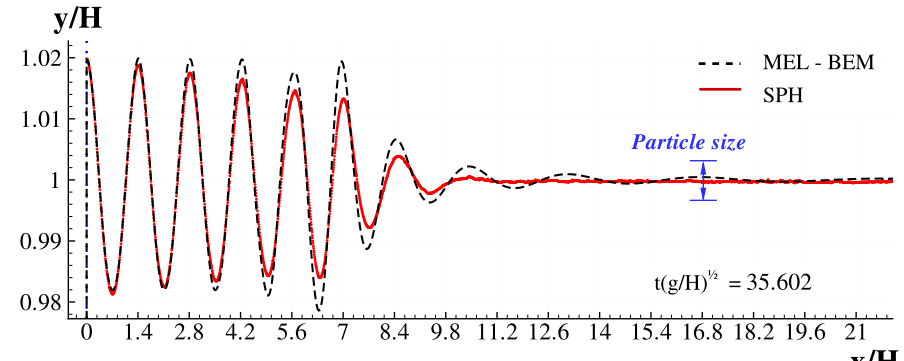

$\mathbf{x} / \mathbf{H}$
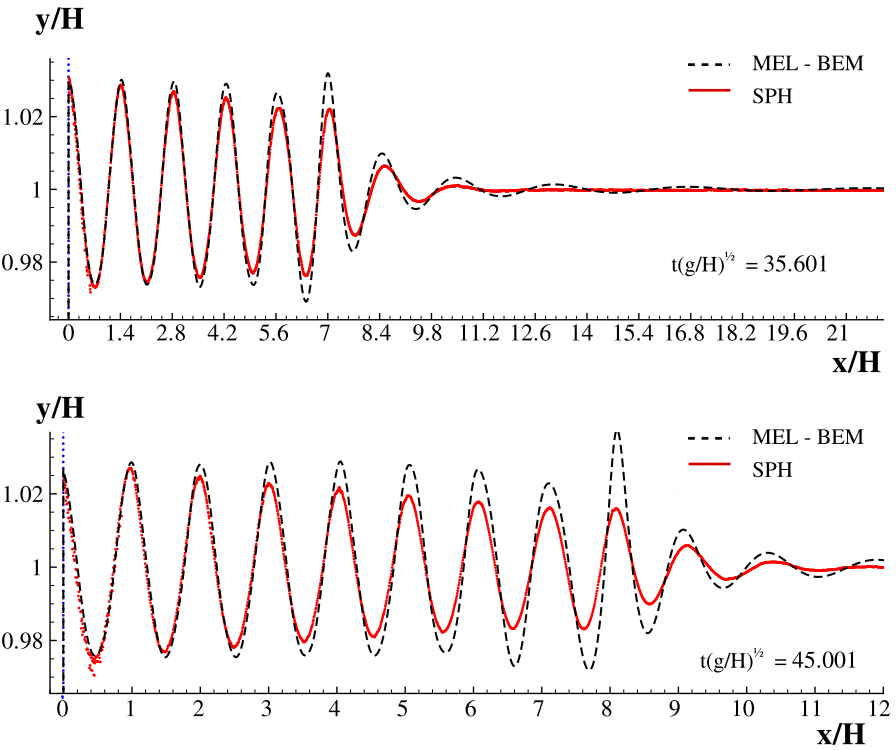

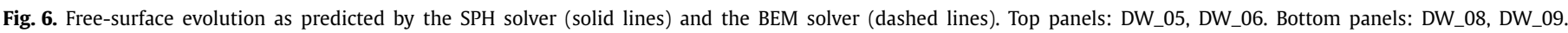
Arrows indicate the particle diameter (the length is referred to the $y$-axis).
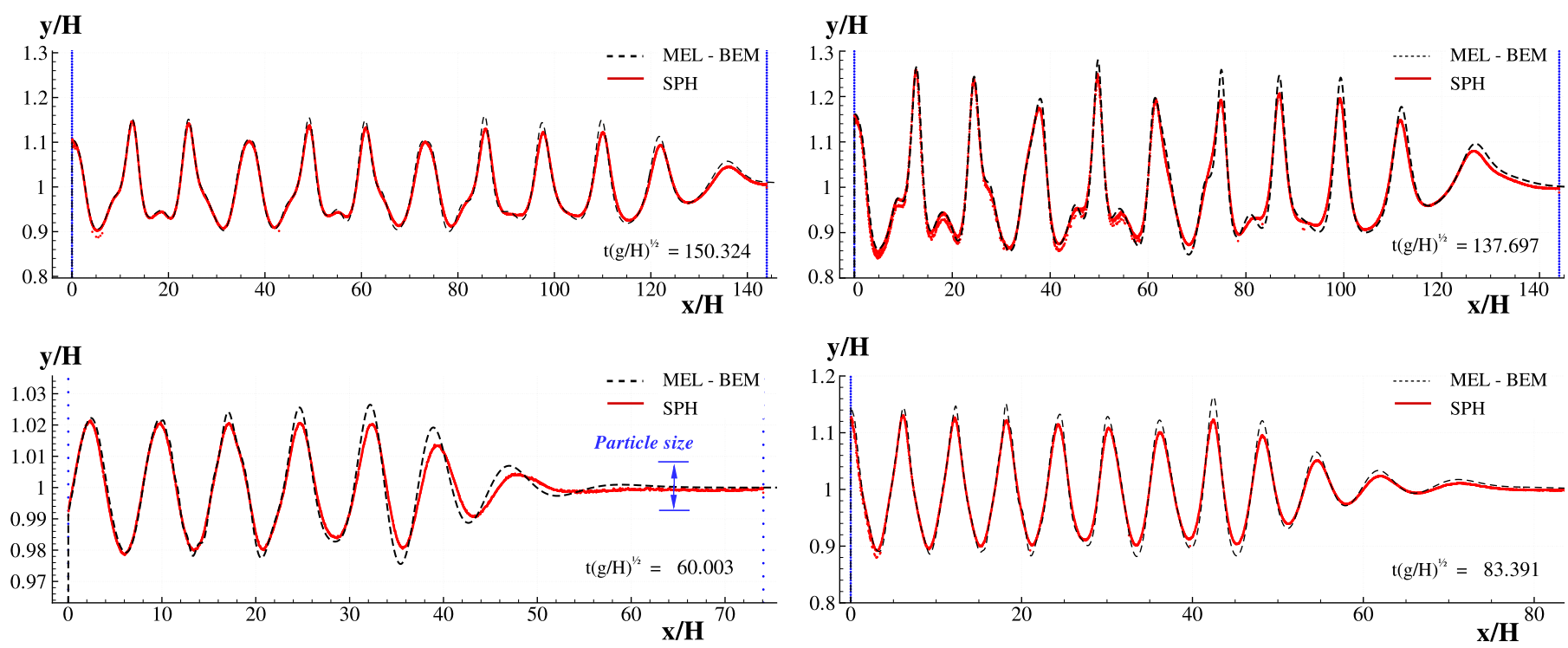

Fig. 7. Free-surface evolution as predicted by the SPH solver (solid lines) and the BEM solver (dashed lines). Top panels: IW_01, IW_02. Bottom panels: IW_03, IW_04.

while the amplitude increases from $\epsilon=0.03$ up to $\epsilon=0.23$. Therefore in these four cases we move from an almost linear wave (DW_01) to a highly nonlinear wave (DW_04). In the latter case, the steepness is quite large (i.e. $\gamma=0.36$ ) leading to the breaking of the first crest at a distance of about $13 \mathrm{H}$ away from the wave maker (see bottom right plot of Fig. 5). The BEM-MEL solver is not able to simulate such a quite steep wave while for the first three cases the match between SPH and BEM-MEL is generally very good: both wave shape and wave celerity are fairly well reproduced and only small damping is observed in the SPH simulations at the wave front. The relative errors for these cases are $\varepsilon_{R}=0.27,0.19,0.17$ respectively (see Appendix B for more details).

In the bottom panels of Fig. 6 we show the comparison $\delta$-SPH versus BEM-MEL for the deep-water cases with the smallest wave length $(\lambda / H=1$, see Table 1$)$. For such cases the effect of the SPH dissipation is quite evident because of the large value of the parameter $\beta T$.
Figs. 7 and 8 show the most significant intermediate- and shallow-water test cases. The overall agreement between the $\delta$ SPH and BEM results is fairly good. The cases IW_02 and SW_06 having the largest ratios $\lambda / d x$ and $A / d x$ show a good agreement with the BEM-MEL solution. Incidentally, we underline that for the propagation of shallow water waves it is generally important to account for the action of boundary layer at the tank bottom. Anyway, the BEM solver cannot implement a no-slip condition along the solid boundary because it is based on the potential theory. Consequently, the influence of the boundary layer at the tank bottom has been neglected and a free-slip condition has been implemented in the SPH solver as well.

Plots in Fig. 9 show the same test case (that is, SW_01) using two different spatial resolutions $(H / d x=20, A / d x=1.2$, top; $H / d x=40, A / d x=2.4$, bottom). For the smallest spatial resolution small oscillations associated with the weakly compressibility appear. This effect disappears when a larges resolution (i.e. $H / d x=40$ ) is used. In Figs. 6, 7, 8 and 9 arrows indicate the particle diameter (the length is referred to the $y$-axis) and prove that 

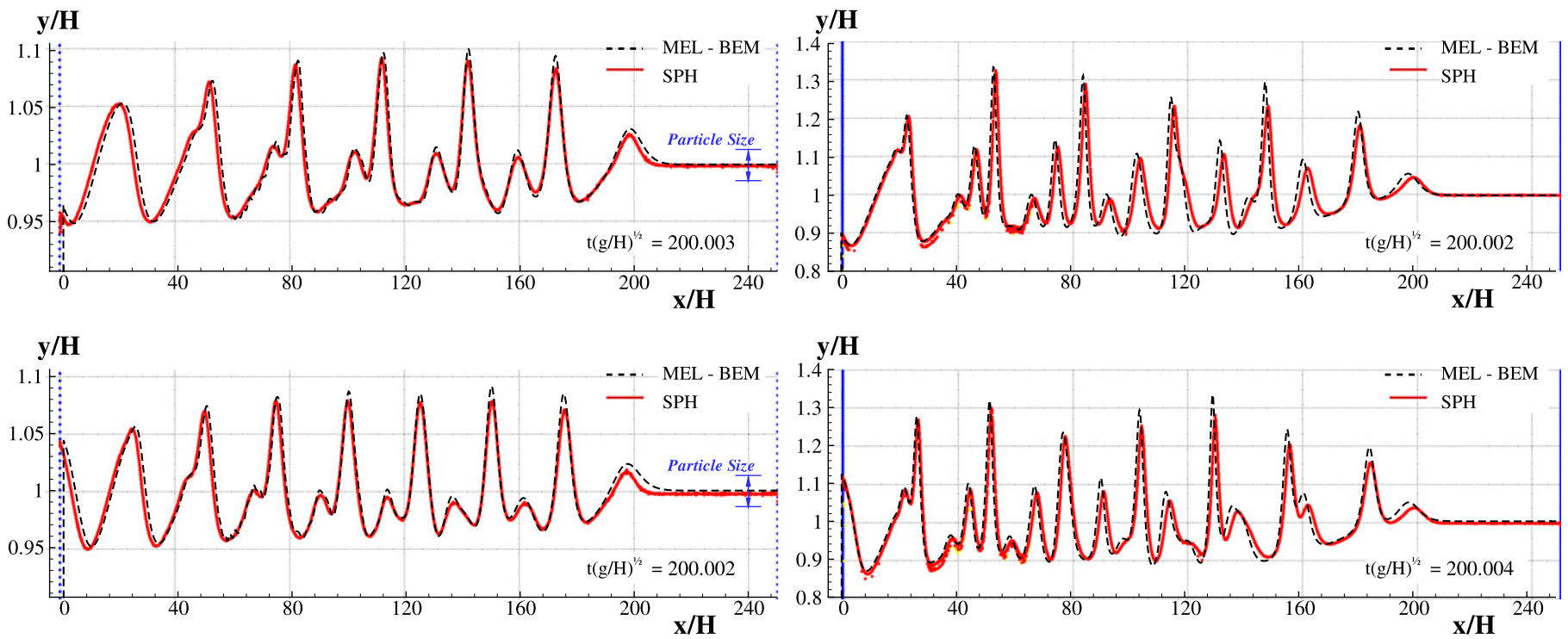

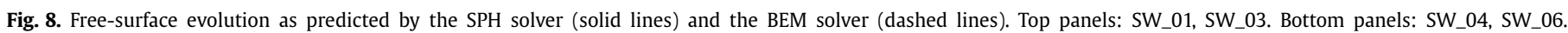
Arrows indicate the particle diameter (the length is referred to the $y$-axis).
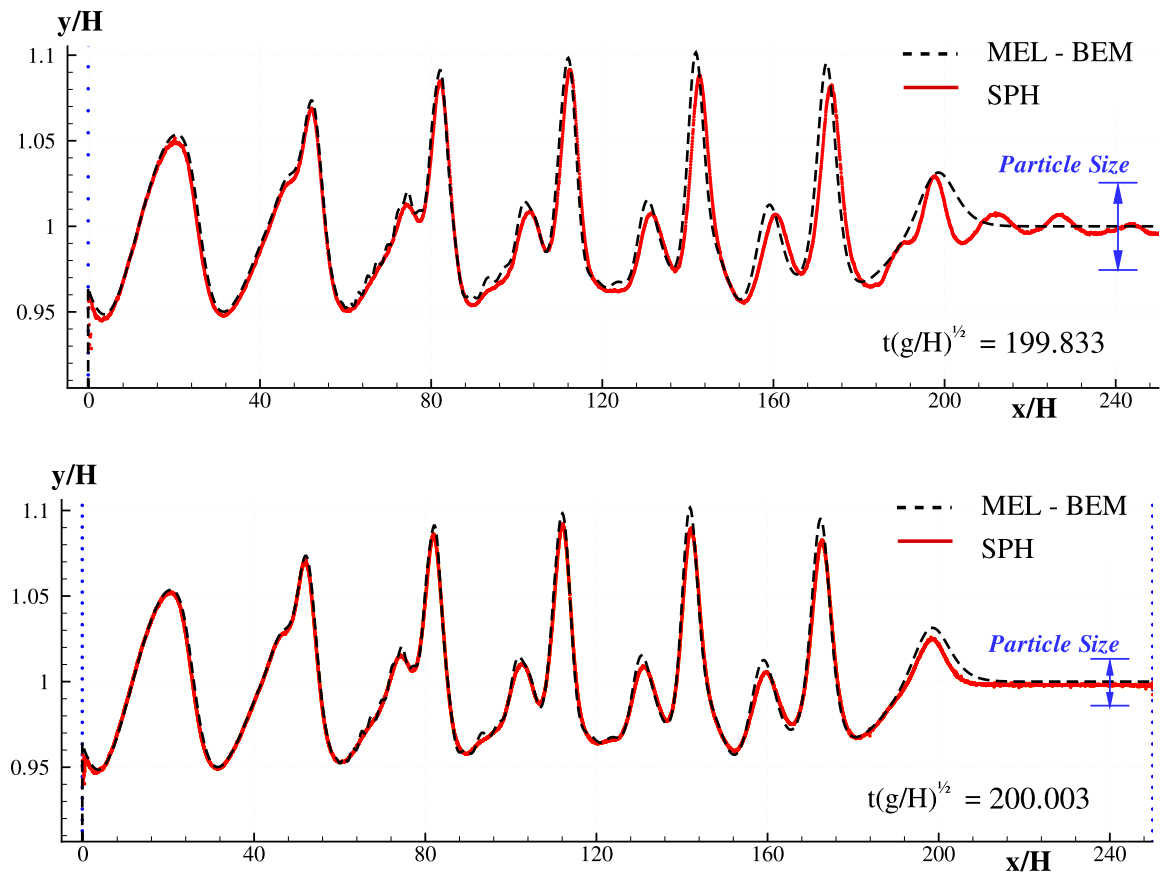

Fig. 9. Test case SW_01. Comparison between two different spatial resolutions: top panel $H / d x=20$, bottom panel $H / d x=40$.

the spurious dissipative effects become smaller for the cases with the largest ratio $A / d x$.

In Fig. 10 we show the convergence analysis of the wave elevation as predicted by the $\delta$-SPH scheme. The test case is the IW_04 and the wave elevation has been recorded at $x / H=18$ using three different spatial discretizations. The convergence rate of the $\mathrm{SPH}$ is about $C=1.5$ (see Appendix B for more details) and the SPH solution tends to the convergent solution of the BEM-MEL signal.

Finally, we conclude this section reporting the computational costs of the $\delta$-SPH scheme for the simulations of the regular waves. For all these cases the same speed of sound has been used, that is $c_{0}=10 \sqrt{g H}$. The time steps of the SPH scheme can be estimated by the equation: $d t=3.5 d x / c_{0}$, using a fourth order Runge-Kutta scheme as time integrator. Since neither breaking wave phenomena nor impacts on solid structures are present, the SPH time step remains almost constant during the simulations. Therefore, to simulate a physical time evolution of $t=100 \sqrt{\mathrm{H} / \mathrm{g}} \mathrm{s}, 286(\mathrm{H} / \mathrm{dx})$ time iterations are needed. The algorithm implemented for the $\delta$-SPH scheme requires a CPU cost of about $30 \mu$ s for each time iteration and for each particle using a single $2.33 \mathrm{GHz}$ XEON core. Using the above estimations the case DW_07 requires 13000 time iterations and takes a CPU time of 57 hours on a single core. The memory occupation is about 500 Mbyte using double precision variables. The same case takes about two hours using the BEM-MEL solver requiring 9000 time steps while the memory requirement is 35 Mbyte. This clearly shows that an optimal choice for an efficient analysis of the gravity waves is to couple the SPH scheme with a potential flow solver. Indeed, the former scheme would be used only where wave breaking can occur while the latter one would be used in the remaining part of the fluid domain. 


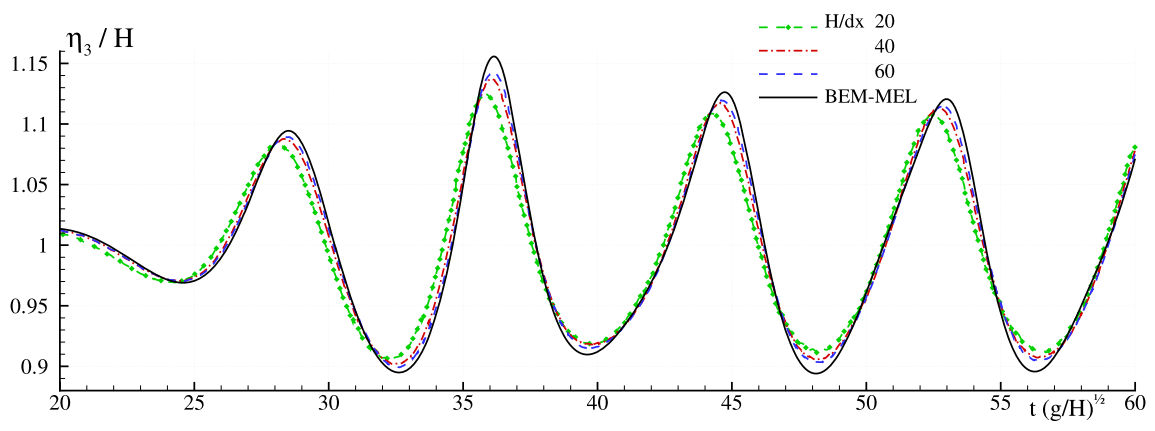

Fig. 10. Convergence analysis for the time history of the wave elevation recorded at $x / H=18$. Test case IW_04.

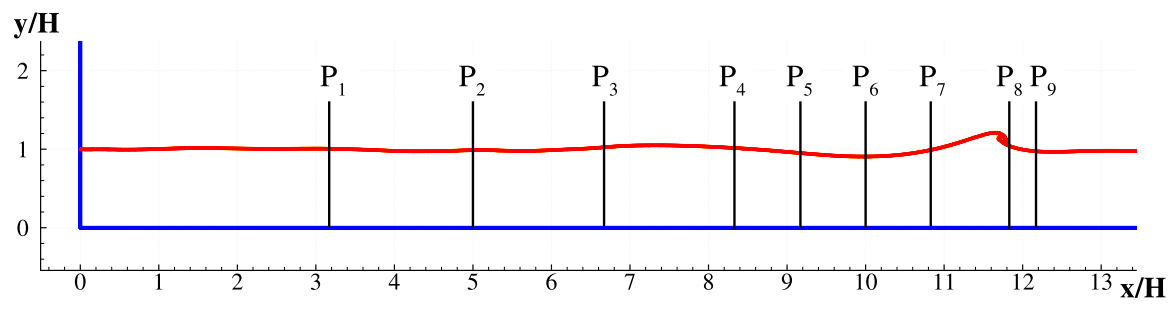

Fig. 11. The basin used in the work by Dommermuth et al. [7]. Probe positions are: $x / H=3.16,5.00,6.66,8.33,9.16,10,10.83,11.83,12.16$.
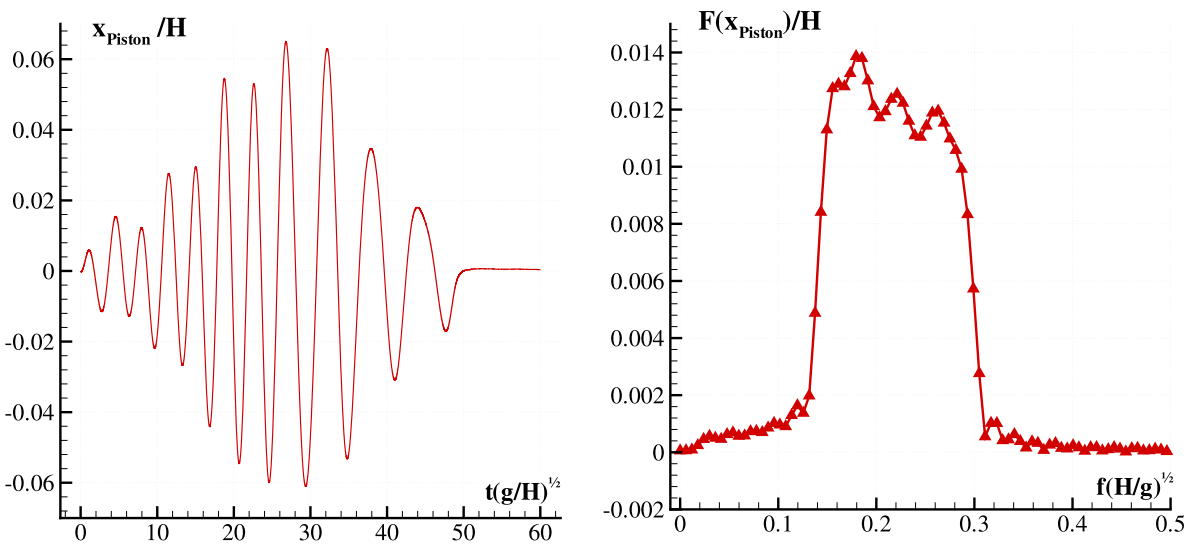

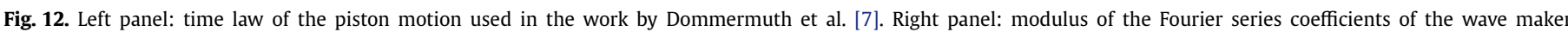
motion.

\subsection{Wave packet}

Having shown the capability of the SPH solver to reproduce the evolution of the regular wave system, in the following the wave packets evolution is used to stress further the ability of the algorithm to deal with the wave propagation problem. The wave packet proposed by Dommermuth et al. [7] is used to get a plunging breaking wave. In Fig. 11 a sketch of the basin used for their experiments is depicted. The wave packet is generated by a linear superposition of wave frequency components with suitable amplitude and phase to get their superposition at a prescribed distance from the wave maker. The point where the several components focus is called focusing point. The left plot of Fig. 12 shows the time history of the piston motion. As shown in the right panel of the same figure, this signal contains several harmonic components in the dimensionless frequency range $(0.15: 0.30)$. To proper capture the highest one, a fine enough spatial discretization is required to avoid the spurious dissipative effects of the SPH scheme.

Along the basin there are nine probes which measure the wave elevation (see Fig. 11). Specifically, probes $P_{8}$ and $P_{9}$ are placed near the breaking point. The comparison with the experimental measurements in Fig. 13 shows an overall good agreement except for a small disturbance observed in the SPH signals at probes $P_{8}$ and $P_{9}$. In the low right panel of the same figure, the convergence analysis for the SPH free surface is displayed for a detail of the signal at probe $P_{8}$. The convergence rate obtained using the resolutions $H / d x=25,50,100$ is $C=1.3$ while for $H / d x=50,100$, 200 we get $C=1.1$. Note that the time signals have been considered up to $t=51 \sqrt{\mathrm{H} / \mathrm{g}}$ since for longer times wave breaking occurs. This analysis is repeated for a snapshot of the free surface evolution near the focusing point (see Fig. 14). The SPH solutions at different spatial resolutions are compared with the BEM solution. The relative errors with respect to the BEM-MEL solver are $\varepsilon_{R}=0.41,0.24,0.15,0.13$ respectively. All figures clearly show that a fine enough resolution is needed to avoid the underestimation of the wave height.

Finally, Fig. 15 compares the free surface predicted by both the BEM-MEL solver and the $\delta$-SPH with the experimental measurements at the plunging point (probe $P_{8}$ ). Both the numerical solvers are in good agreement even if they tend to anticipate the experimental data. The small delay of the BEM-MEL solver with respect to the $\delta$-SPH is due to the use of a regridding algorithm. 

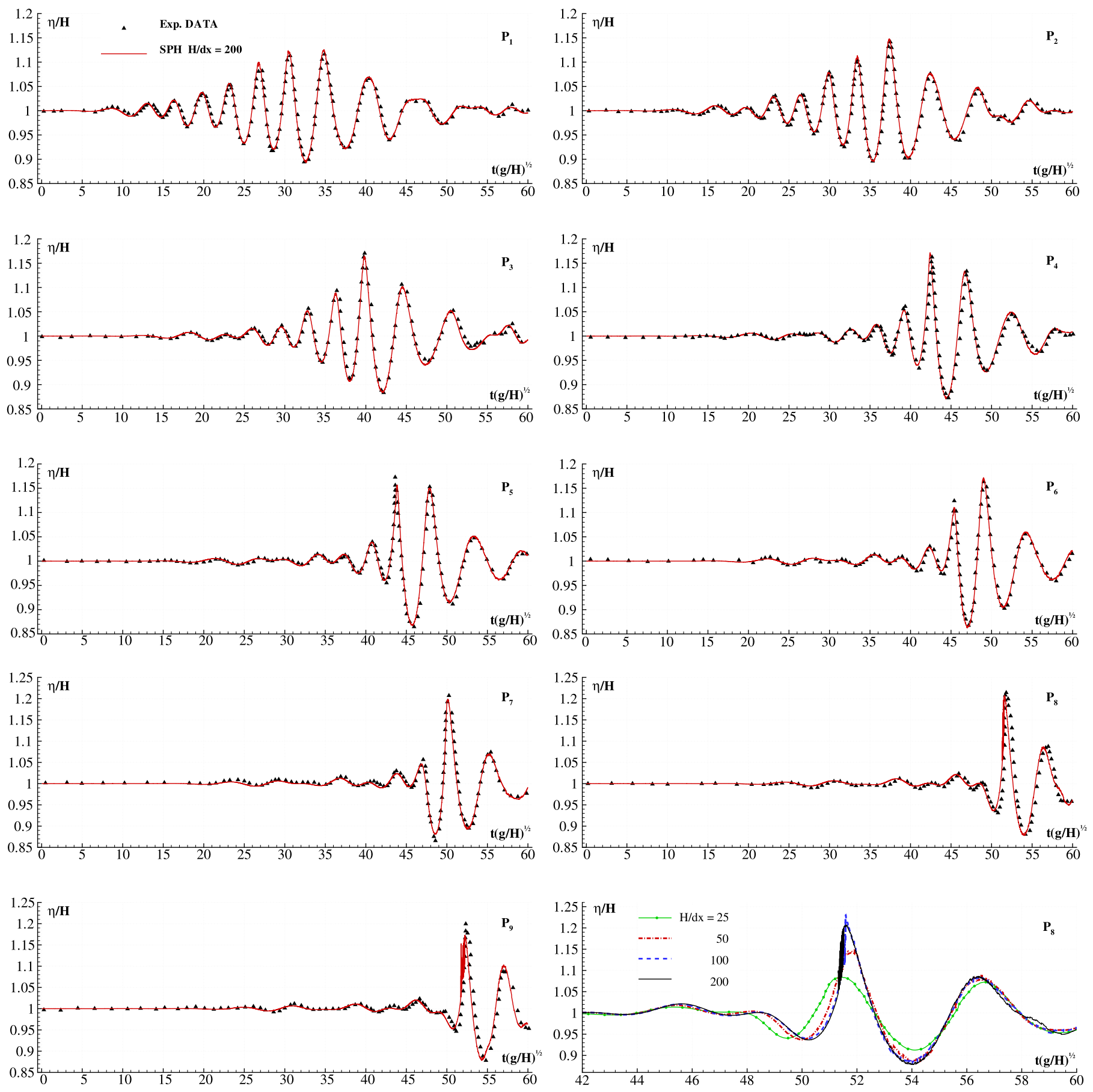

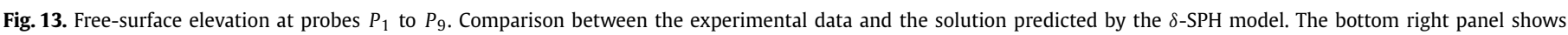
the convergence analysis at probe $P_{8}$ ( $H$ is the still water depth and $d x$ is the mean particle distance).

Such a numerical technique is widely used inside the numerical solvers based on the potential theory (see for example Dommermuth et al. [7]) to remove the numerical instabilities of the freesurface.

During the post breaking evolution, the BEM-MEL solver is unable to follow this stage since vorticity is generated consequently to the cavity closure. In contrast, the SPH method can simulate this phenomenon and in Fig. 15 the vorticity generated after the plunging of the breaking wave is displayed. Specifically, two vortical structures are induced by the splashing processes similarly to what shown in [14]. Since both positive and negative vorticities are generated, dipole structures are formed which tend to move downwards away from the free surface.

\section{Conclusions}

The ability of the $\delta$-SPH solver to properly model the wave propagation phenomenon has been inspected. This model, defined as a simplified version of the one proposed in Antuono et al. [1], is associated with the enhanced treatment of the solid boundary described in Colagrossi et al. [3]. Standing waves, regular waves and wave packet have been studied in details and compared respectively with the approximate analytical solution by Lighthill [17], with a BEM-MEL solver [20] and with the experimental measurements by Dommermuth et al. [7]. In all the cases considered, the $\mathrm{SPH}$ proved able to reproduce the propagation of gravity waves in all the regimes (i.e. from deep to shallow water) showing a 

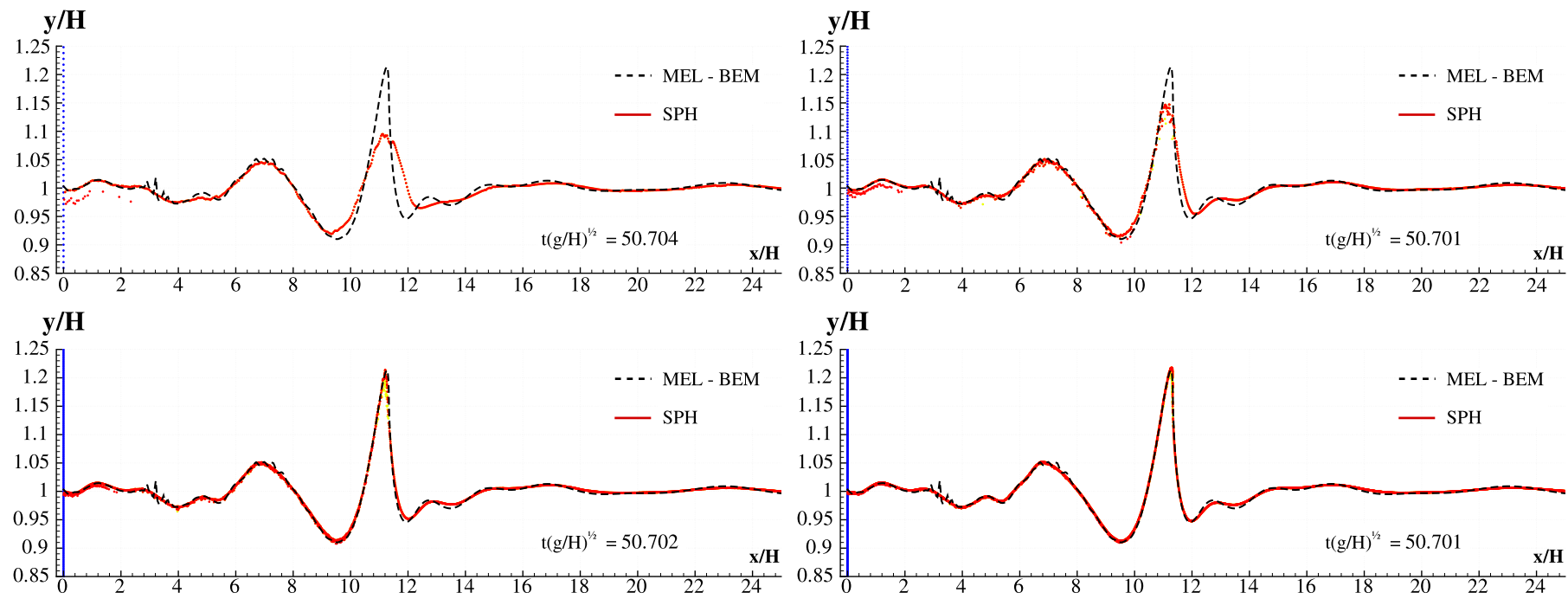

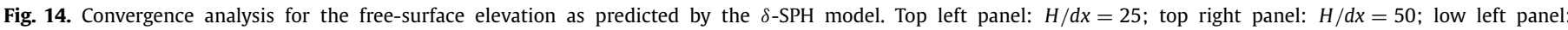
$H / d x=100$; low right panel: $H / d x=200$ ( $H$ is the still water depth and $d x$ is the mean particle distance).
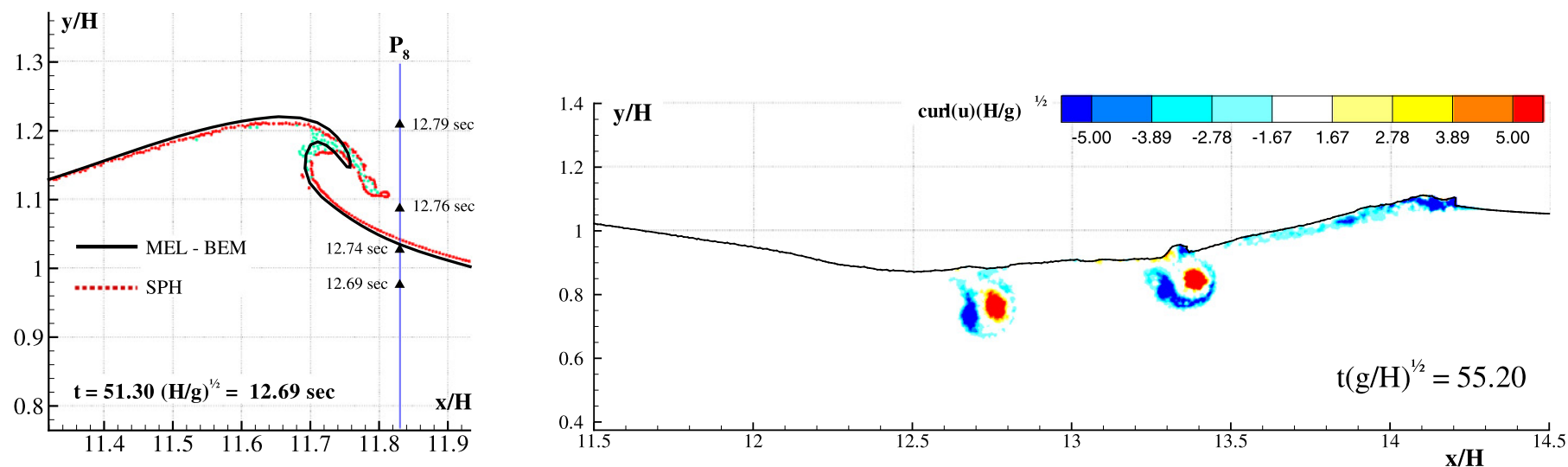

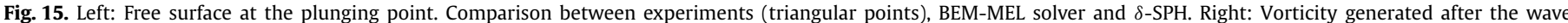
breaking.

good overall agreement with analytical, numerical and experimental data. Finally, the present study allowed understanding which is the minimal spatial resolution needed to properly model the propagation phenomenon and avoid an overdamping of the gravity wave signals.

\section{Acknowledgements}

The research leading to these results has received funding from the European Community's Seventh Framework Programme (FP7/2007-2013) under grant agreement No. 225967 "NextMuSE". This work was also partially supported by the Centre of Excellence for Ship and Ocean Structures of NTNU Trondheim (Norway) within the "Violent Water-Vessel Interactions and Related".

\section{Appendix A. The wave maker motion}

The time law of the piston is given by:

$x_{w}(t)=\frac{\Delta x}{2} \sin (\omega t)$,

where $x_{w}$ is the $x$-coordinate of the wave maker and $\Delta x / 2$ is its maximum displacement from the position of rest. For a flap it is:

$x_{w}(t, z)=\frac{\Delta x}{2}\left[1-\frac{z}{H-h_{f}} \sin (\omega t)\right]$, where $h_{f}$ is the position of the hinge from the tank bottom. In some test cases, a ramp is used to avoid impulsive transitory. This is given by a function $R(t)$ which multiplies $x_{w}(t)$. This function is given below:

$R(t)=\frac{1}{2}\left[1-\cos \left(\pi \frac{t}{\tau}\right)\right]$

where $\tau$ is a fixed time interval which corresponds to the ramp duration. All the details on the wave maker and on the ramp are summarized in Table 4.

\section{Appendix B. Convergence analysis: details of computations}

In this brief section we report some details on the convergence analysis.

Let us consider the signal $f=\eta(s)-H$ with $s \in\left[s_{0}, s_{f}\right]$. Here, the symbol $s$ represents the spatial or the time variable according to the case at hand. Then, the error between two signals $f_{1}$ and $f_{2}$ in the $L^{1}$-norm is:

$\varepsilon_{21}=\int_{s_{0}}^{s_{f}}\left|f_{1}-f_{2}\right| d s$. 
Table 4

Regular waves. Letters 'P' and ' $\mathrm{F}$ ' indicate respectively that a piston or a flap is used.

\begin{tabular}{|c|c|c|c|c|}
\hline & WM & $\Delta x / H$ & $h_{f} / H$ & $\tau \sqrt{g / H}$ \\
\hline SW_01 & $\mathrm{P}$ & 0.50 & 1 & 1 \\
\hline SW_02 & $\mathrm{P}$ & 1.00 & 1 & 1 \\
\hline SW_03 & $\mathrm{P}$ & 1.30 & 1 & 15.00 \\
\hline SW_04 & $\mathrm{P}$ & 0.40 & I & 1 \\
\hline SW_05 & $\mathrm{P}$ & 0.70 & 1 & 1 \\
\hline SW_06 & $\mathrm{P}$ & 1.10 & 1 & 12.00 \\
\hline SW_07 & $\mathrm{P}$ & 0.40 & 1 & 1 \\
\hline SW_08 & $\mathrm{P}$ & 0.70 & 1 & 1 \\
\hline SW_09 & $\mathrm{P}$ & 0.90 & I & 10.00 \\
\hline IW_01 & $\mathrm{P}$ & 0.400 & i & 6.00 \\
\hline IW_02 & $\mathrm{P}$ & 0.600 & 1 & 6.00 \\
\hline IW_03 & $\mathrm{P}$ & 0.052 & i & 2.13 \\
\hline IW_04 & $\mathrm{P}$ & 0.264 & 1 & 2.13 \\
\hline IW_05 & $\mathrm{F}$ & 0.200 & 0.0 & 3.00 \\
\hline IW_06 & $\mathrm{F}$ & 0.400 & 0.0 & 3.00 \\
\hline IW_07 & $\mathrm{F}$ & 0.102 & 0.0 & 4.00 \\
\hline IW_08 & $\mathrm{F}$ & 0.200 & 0.0 & 4.00 \\
\hline DW_01 & $\mathrm{F}$ & 0.025 & 0.0 & 5.00 \\
\hline DW_02 & $\mathrm{F}$ & 0.050 & 0.0 & 5.00 \\
\hline DW_03 & $\mathrm{F}$ & 0.100 & 0.0 & 5.00 \\
\hline DW_04 & $\mathrm{F}$ & 0.200 & 0.0 & 5.00 \\
\hline DW_05 & $\mathrm{F}$ & 0.028 & 0.3 & 4.00 \\
\hline DW_06 & $\mathrm{F}$ & 0.042 & 0.3 & 4.00 \\
\hline DW_07 & $\mathrm{F}$ & 0.010 & 0.5 & 3.00 \\
\hline DW_08 & $\mathrm{F}$ & 0.020 & 0.5 & 3.00 \\
\hline DW_09 & $\mathrm{F}$ & 0.040 & 0.5 & 3.00 \\
\hline
\end{tabular}

Now, let assume that $f_{2}$ is obtained by doubling the resolution of $f_{1}$ and, similarly, consider a signal $f_{3}$ that doubles the resolution of $f_{2}$. Then, the convergence rate of the quantity $f$ is given by:

$C=\log \left(\frac{\varepsilon_{32}}{\varepsilon_{21}}\right) / \log (2)$.

The relative error with respect to the BEM solutions is given by:

$\varepsilon_{R 1}=\left(\int_{s_{0}}^{s_{f}}\left|f_{1}-f_{B}\right| d s\right)\left(\int_{s_{0}}^{s_{f}}\left|f_{B}\right| d s\right)^{-1}$

where the symbol $f_{B}$ indicates the BEM signal, here regarded as the reference solution (that is, as an "exact" solution).

\section{References}

[1] M. Antuono, A. Colagrossi, S. Marrone, D. Molteni, Free-surface flows solved by means of SPH schemes with numerical diffusive terms, Comput. Phys. Comm. 181 (3) (2010) 532-549.

[2] A. Colagrossi, M. Landrini, Numerical simulation of interfacial flows by smoothed particle hydrodynamics, J. Comput. Phys. 191 (2003) 448-475.
[3] A. Colagrossi, M. Antuono, S. Marrone, A 2D+t SPH model with enhanced solid boundary treatment, in: Proc. of 4th International SPHERIC Workshop, Nantes, France, May 2009.

[4] A. Colagrossi, M. Antuono, D. Le Touzé, Theoretical considerations on the free surface role in the SPH model, Phys. Rev. E 79 (5) (2009) 1-13 (056701).

[5] J.W. Dold, An efficient surface-integral algorithm applied to unsteady gravity waves, J. Comput. Phys. 03 (1992).

[6] J.W. Dold, D.H. Peregrine, Water-wave modulation, in: Proc. 20th Int. Conf. on Coastal Eng., vol. I, 1986.

[7] D.G. Dommermuth, D.K.P. Yue, W.M. Lin, R.J. Rapp, Deep-water plunging breakers: a comparison between theory and experiments, J. Fluid Mech. 189 (1988) 423-442.

[8] M. Doring, Développement d'une méthode SPH pour les applications à surface libre en hydrodynamique, PhD thesis, Ecole Centrale Nantes, 2005.

[9] P. Español, M. Revenga, Smoothed dissipative particle dynamics, Phys. Rev. 67 (2003).

[10] A. Ferrari, M. Dumbser, E.F. Toro, A. Armanini, A new 3D parallel SPH scheme for free-surface flows, Comput. \& Fluids 38 (2009) 1203-1217.

[11] T.P. Fries, H.G. Matthies, Classification and overview of meshfree methods, Informatikbericht 2003-03, Institute of Scientific Computing, Technical University Braunschweig, Brunswick, Germany, 2004.

[12] S.T. Grilli, I.A. Svendsen, Corner problems and global accuracy in the boundary element solution of nonlinear wave flows, Eng. Anal. Bound. Elem. 7 (4) (1990).

[13] P.M. Guilcher, Contribution au developpement d'une methode SPH pour la simulation numerique des interactions houle-structuré, PhD thesis, ECN, Nantes, 2008.

[14] M. Landrini, A. Colagrossi, M. Greco, M.P. Tulin, Gridless simulations of splashing processes and near-shore bore propagation, J. Fluid Mech. 591 (2007) 183213.

[15] N. Lanson, J.P. Vila, Meshless methods for conservation laws, Math. Comput. Simulation 55 (2001) 493-501.

[16] P.W. Randles, L.D. Libersky, Smoothed particle hydrodynamics: Some recent improvements and applications, Comput. Methods Appl. Mech. Engrg. 139 (1996) 375-408.

[17] J. Lighthill, Waves in Fluids, Cambridge University Press, New York, 2001.

[18] M.S. Longuet-Higgins, E.D. Cokelet, The deformation of steep surface waves on water. Part I. A numerical method of computation, Proc. R. Soc. Lond. A 350 (1976) 1-26.

[19] M.S. Longuet-Higgins, Breaking waves in deep and shallow water, in: Proc. 10th Conf. on Naval Hydrodynamics, Boston, 1976.

[20] C. Lugni, A study about the free-surface waves and the freely floating structure interactions, PhD thesis, University of Rome "La Sapienza", 1999 (in Italian).

[21] S. Marrone, M. Antuono, A. Colagrossi, G. Colicchio, G. Graziani, Enhanced boundary treatment in 2D smoothed particle hydrodynamics models, in: Proc. XIX Congress AIMETA, Ancona, Italy, 2009.

[22] P.A. Madsen, H.A. Shaffer, A discussion of artificial compressibility, Coast. Eng. J. 53 (2006) 93-98.

[23] S. Marrone, A. Colagrossi, D. Le Touzé, G. Graziani, Fast free-surface detection and level-set function definition in SPH solvers, J. Comput. Phys. 229 (2010) 3652-3663.

[24] D. Molteni, A. Colagrossi, A simple procedure to improve the pressure evaluation in hydrodynamic context using the SPH, Comput. Phys. Comm. 180 (6) (2008) 861-872.

[25] J.J. Monaghan, Smoothed particle hydrodynamics, Rep. Progr. Phys. 68 (2005) 1703-1759.

[26] P. Wang, Y. Yao, M.P. Tulin, An efficient numerical wave tank for nonlinear water waves, based on the multi-subdomain approach with BEM, Internat. J. Numer. Methods Fluids 20 (1995). 